Pacific Journal of Mathematics

THE GROUP OF SELF-EQUIVALENCES OF CERTAIN

COMPLEXES 


\section{THE GROUP OF SELF-EQUIVALENCES OF CERTAIN COMPLEXES}

\section{DAVID SMALLEN}

The group of self-equivalences (homotopy classes of base point preserving homotopy equivalences) of a certain class of finite $C W$-complexes is studied. This class includes, in particular, all closed, connected, $n$-manifolds $M$ with finite fundamental group such that $\pi_{i}(M)=0,1<i<n$. Such complexes are easily seen to be the quotient space of a fixed point free action of a finite group on a homotopy $n$-sphere, and include the Klein-Clifford manifolds.

The main result characterizes this gromp as a normal subgroup of Aut $\left(\pi_{1}(X)\right)$, for $X$ in the above mentioned class, consisting of all $\theta$ such that $\theta$ induces either the identity map or the inverse map on $H^{n+1}\left(\pi_{1}(X) ; Z\right)=Z_{k}$, $k$ being the order of $\pi_{1}(X)$. This leads to a collection of general results on the algebraic structure of the group of self-equivalences, as well as several explicit calculations, including the recovery of results due to Olum.

This group has been studied by various authors, e.g. ArkowitzCurjel [1], Olum [7], Rutter [8], and Shih [9]. In general this group is non-abelian and quite often infinite.

Let $\Sigma^{n}$ denote a finite $n$-dimensional $C W$-complex of the homotopy type of an $n$-sphere. Let $G$ be a finite group which acts without fixed points on $\Sigma^{n}$ and let $X=\Sigma^{n} / G$ denote the quotient space of $\Sigma^{n}$ by the action of $G$. We study the group of self-equivalences of $X$.

Several comments are in order at this point. Firstly, if the order of $G$ is one or two the results are well known and although our methods apply we omit discussion of these in order that our results be more succintly stated. Secondly, if the order of $G$ is greater than two (the case we study) $n$ must be odd. Finally, it is easily shown, e.g. [10], that $\pi_{1}(X)$ acts trivially on $\pi_{j}(X), j>1$. We point out that $G$ may often be non-abelian but $G$ must be periodic of period $n+1$ (see [2], p. 260).

We define the group of seli-equivalences for completeness.

Let $Y$ be a topological space with base point $y \in Y$. Let $\mathscr{H}(Y)$ denote the collection of all homotopy equivalences of $Y$ preserving base point. The equivalence relation $\sim$ of base point preserving homotopy divides $\mathscr{H}(Y)$ into classes called self-equivalences of $Y$ and we bave 
Definition. $E q(Y)=\mathscr{H}(Y) / \sim . E q(Y)$ is a group under the operation induced by composition of mappings.

We note first that

Lemma 1.1. $E q(X)$ is isomorphic to $E q\left(X_{n+1}\right)$ where $X_{n+1}$ is the $(n+1)$ st stage of the Postnikov system of $X$.

Proof. This follows from the fact that $\pi_{1}(X)$ acts trivially on $\pi_{j}(X), j>1$ and a simple obstruction theory argument as in [1] where the result is stated in the simply connected case.

The Postnikov system for $X$ begins as follows,

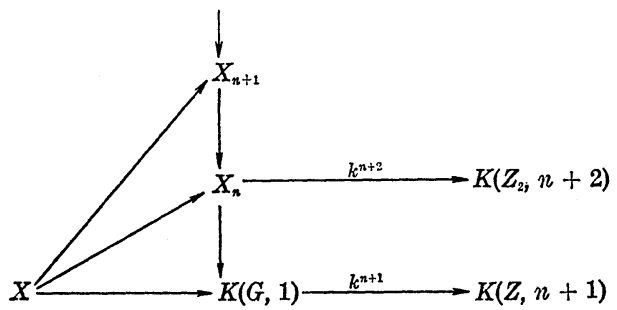

FIGURE 1

As was pointed out above $n$ must be odd so $\pi_{n+1}(X) \approx \pi_{n+1}\left(\Sigma^{n}\right)=$ $Z_{2}$.

We define $\psi_{G}=\left\{\theta \in[K(G, 1), K(G, 1)] \mid \theta^{*}\left(k^{n+1}\right)= \pm k^{n+1}\right\}$. Then

LemMA 1.2. $E q\left(X_{n}\right) \approx \psi_{G}$.

Proof. By Shih [9] we have the exact sequence

$$
1 \longrightarrow H^{n}(K(G, 1) ; Z) \longrightarrow E q\left(X_{n}\right) \longrightarrow \psi_{G} \longrightarrow 1 \text {. }
$$

Now since $G$ acts without fixed points on $\Sigma^{n}$ and hence is periodic, the odd dimensional cohomology of $G$ vanishes, so the result follows. Now we show that

LEMma 1.3. $E q\left(X_{n+1}\right) \approx E q\left(X_{n}\right)$.

Proof. By Kahn [3] we have homomorphism $\rho^{n}: E q\left(X_{n+1}\right) \rightarrow$ $E q\left(X_{n}\right)$. By a Serre spectral sequence argument one can easily show that $H^{n+2}\left(X_{n}: Z_{2}\right)=Z_{2}$ and hence if $f \in E q\left(X_{n}\right), f^{*}: H^{n+2}\left(X_{n}, Z_{2}\right) \rightarrow$ $H^{n+2}\left(X_{n}: Z_{2}\right)$ is the identity map. This implies that $\rho^{n}$ is onto by Lemma 2.1 of [3].

Now in the paper cited it is also shown that $\operatorname{Ker} \rho^{n}$ is the image of a subgroup of $H^{n+1}\left(X_{n+1} ; Z_{2}\right)$. Again using a Serre spectral 
sequence argument one can show $H^{n+1}\left(X_{n+1} ; Z_{2}\right)=0$. Hence the result follows.

Collecting together the proceeding we have

THeOREM 1.4. If $X$ is as described above then $E q(X)$ is isomorphic to $\left\{\theta \in\right.$ Aut $\left(\pi_{1}(X)\right) \mid \theta^{*}$ is either the identity map on $H^{n+1}\left(\pi_{1}(X) ; Z\right)$ or the map which takes each element to its algebraic inverse $\}$. Here $H^{*}\left(\pi_{1}(X) ; Z\right)$ denotes the group cohomology of $\pi_{1}(X)$, see [12].

Proof. We note firstly that by the periodicity of $G$ we have $H^{n+1}\left(\pi_{1}(X) ; Z\right) \approx Z_{m}$ where $m$ is the order of $G$. Secondly $k^{n+1}$ is easily seen to be a generator of the group by a spectral sequence argument. Finally it can be shown (see Maclane [4], p. 136) that the cohomology of a $K(G, 1)$ space is "naturally" isomorphic to the "group cohomology" $H^{*}(G ; Z)$. The naturality of this isomorphism means in particular that if $h$ is a map from $K(G, 1)$ to itself inducing $\theta$ on $\pi_{1}(K(G, 1))=G$ then the following diagram commutes where $\Psi$ is the natural isomorphism.

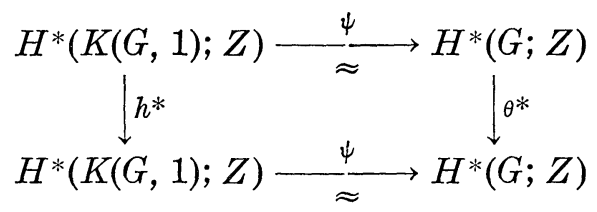

Figure 2

Hence the problem is reduced to a purely (cohomological) algebraic criterion. We see in the remainder of this section and in $\S 2$ that this theorem quite often is a computational tool in determining $E q(X)$.

First we note immediately that

COROLlary 1.5. $E q(X)$ is a normal subgroup of Aut $\left(\pi_{1}(X)\right)$.

Proof. Let $\nu \in \operatorname{Aut}\left(\pi_{1}(X)\right)$. Then if $\theta \in E q(X)$ (here we identify $E q(X)$ with the elements of the automorphism group), we show $\nu \cdot \theta \cdot \nu^{-1} \in E q(X)$. Let $k \in H^{n+1}(G ; Z)$. Then

$$
\left[\nu \cdot \theta \cdot \nu^{-1}\right]^{*} k=\nu^{-1 *} \theta^{*}\left[\nu^{*} k\right]=\nu^{-1 *}\left( \pm \nu^{*} k\right)= \pm k \text {. }
$$

CoRollary 1.6. $E q(X) \supseteqq \vartheta n n\left(\pi_{1}(X)\right)$, the group of inner automorphisms of $\pi_{1}(X)$.

Proof. All inner automorphisms have the property that they 
induce the identity map on cohomology ([4], p. 118).

This is particularly useful since a standard group theory fact is that $\ln n(G) \approx G / Z(G), Z(G)$ the center of $G$.

Corollary 1.7. $E q(X)$ is solvable if and only if Aut $\left(\pi_{1}(X)\right)$ is solvable.

Proof. Since $E q(X)$ is normal in Aut $\left(\pi_{1}(X)\right)$ the result will follow if we can show Aut $\left(\pi_{1}(X)\right) / E q(X)$ is solvable. Let

$$
\Psi: \text { Aut }\left(\pi_{1}(X)\right) \longrightarrow \operatorname{Aut}\left(H^{n+1}\left(\pi_{1}(X) ; Z\right)\right)
$$

be the obvious map $\theta \rightarrow \theta^{*}$. Now since $H^{n+1}\left(\pi_{1}(X) ; Z\right)$ is cyclic we have Aut $\left(H^{n+1}\left(\pi_{1}(X) ; Z\right)\right)$ is abelian. $E q(X) \supseteqq \operatorname{Ker} \Psi$ so

$$
\text { Aut }\left(\pi_{1}(X)\right) / E q(X)
$$

is abelian and hence solvable.

The homomorphism $\Psi$ is a very useful one in the general problem of determining $E q(X)$. In fact $E q(X)=\Psi^{-1}(K)$ where $K$ is the subgroup of Aut $\left(Z_{m}\right), m=$ order of $\pi_{1}(X)$, consisting of the identity map and the inverse automorphism $(x \rightarrow-x)$.

We now consider what happens if $G$ and $H$ are finite groups of orders at least 2 and $G \times H$ acts on $\Sigma^{n}$ without fixed points. The quotient space is a complex of the type considered in this paper. We point out that if $G \times H$ acts on $\Sigma^{n}$ without fixed points then $(|G|,|H|)=1$. This follows from the periodicity of $G, H$ and $G \times H$. We have

THEOREM 1.8. If $G \times H$ acts on $\Sigma^{n}$ without fixed points and $|G| \geqq 2,|H| \geqq 2$, then we have the following two cases:

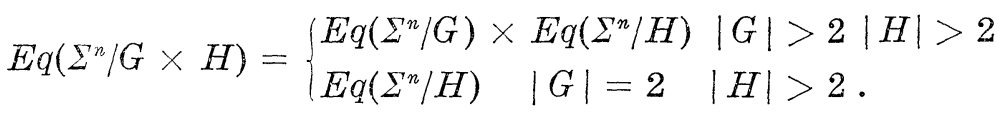

Proof. This follows by looking at the Kunneth theorem in group cohomology and roting that since the orders of $G$ and $H$ are relatively prime Aut $(G \times H) \approx \operatorname{Aut}(G) \times \operatorname{Aut}(H)$.

The situation of a product of two groups acting on $\Sigma^{n}$ occurs frequently (see Milnor [5]). In particular the theorem says that to determine $E q(X)$ one should look at indecomposable groups.

2. Specific calculations of $E q(M)$. In this section we calculate explicitly the group $E q(M)$ for certain manifolds $M$. In particular we recovers results of Olum for generalized Lens spaces, and show 
$E q(X) \neq 0$ for all $X$ studied in this paper. The methods used in the calculations vary, but fall generally in the domain of group theory and cohomology of groups.

A general reference for this section is Weiss [12].

2A. Generalized lens spaces. We now recover results originally due to Olum in [6] by calculating $E q\left(\Sigma^{2 n+1} / Z_{m}\right) n \geqq 1 m>2$. We point out that a fixed-point-free action of a cyclic group on an odd dimensional sphere is always defined, namely to get the generalized Lens spaces. However, our proof does not make use of any "particular" action of $Z_{m}$ on $\Sigma^{2 n+1}$, but in fact shows that $E q\left(\Sigma^{2 n+1} / Z_{m}\right)$ is not dependent on how $Z_{m}$ acts freely, only that it does act in some fixed-point-free way.

We prove:

Theorem 2A. 1. Let $n \geqq 1, m>2$. Then $E q\left(\Sigma^{2 n+1} / Z_{m}\right) \approx \mathscr{Q}_{m}$, where $\mathscr{U}_{m}$ is the subgroup of Aut $\left(Z_{m}\right)$ consisting of those integers $t, 1 \leqq t<m$ such that $t^{n+1} \equiv \pm 1(\bmod m)$. Here we identify Aut $\left(Z_{m}\right)$ with $\{x \mid 1 \leqq x<m,(x, m)=1\}$.

Proof. In this proof we calculate directly the induced map on cohomology of an automorphism $\theta: Z_{m} \rightarrow Z_{m}$ by constructing a chain map on a projective resolution of $Z$ as a trivial $Z\left(Z_{m}\right)$-module (see Weiss [12]).

We take the particularly nice resolution of $Z$ given by

$$
0 \longleftarrow Z \stackrel{d_{0}}{\longleftarrow} \Lambda a_{0} \stackrel{d_{1}}{\longleftarrow} \Lambda a_{1} \stackrel{d_{2}}{\longleftarrow} \Lambda a_{2} \stackrel{d_{3}}{\longleftarrow} \ldots
$$

where $\Lambda=Z\left(Z_{m}\right), Z_{m}$ generated by $\nu, d_{0}$ is the augmentation and

$$
\begin{aligned}
d_{2 k}\left(a_{2 k}\right) & =\left(\nu^{m-1}+\cdots+1\right) a_{2 k-1} \\
d_{2 k+1}\left(a_{2 k+1}\right) & =(\nu-1) a_{2 k}
\end{aligned}
$$

(see Cartan-Eilenberg [2], p. 251).

Let $\theta_{k}: Z_{m} \rightarrow Z_{m}$ be the automorphism given by $\theta_{k}(\nu)=\nu^{k}$, $(k, m)=11 \leqq k<m$. We calculate the induced map on $H^{*}\left(Z_{m} ; Z\right)$ of $\theta_{k}$, by constructing a chain map $\Lambda=\left(\Lambda^{i}\right) i \geqq 0$ corresponding to the pair $\left(\theta_{k}, 1_{z}\right)$ as described in Weiss [12].

By direct calculation we get the diagram

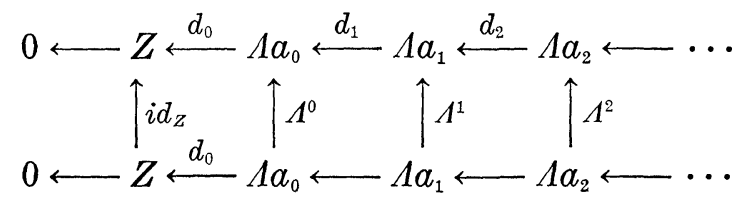

FIGURE 3 
where $\Lambda^{2 i}\left(a_{2 i}\right)=\left[\left(J_{2}\right)^{i}\right] a_{2 i} i \geqq 0$

$$
\Lambda^{2 i+1}\left(a_{2 i+1}\right)=\left[\left(J_{2}\right)^{i} \cdot J_{1}\right] a_{2 i+1}
$$

where

$$
J_{1}=\frac{\left(\nu^{k}-1\right)}{(\nu-1)}, \quad J_{2}=\frac{\left(\nu^{m k}-1\right)}{(\nu-1)}
$$

each considered as elements in $\Lambda$. It can be directly verified that $\left(A^{i}\right) i \geqq 0$, is a chain map (i.e., the above diagram commutes), where we assume the upper resolution is a $\Lambda$-module via the map $\theta_{k}$.

We now wish to determine what the induced map in cohomology will be in dimension $2 n+2$ (since $Z_{m}$ acts on a $2 n+1$-sphere). It is clear that since the coefficients $Z$ in $H^{*}\left(Z_{m}, Z\right)$ are to be regarded as a trivial $\Lambda$-module, that the induced map on cohomology of $\theta_{k}$ is gotten by multiplication by $J_{2}^{n+1}(1)$, where this means evaluating the element $J_{2}^{n+1} \in \Lambda$ by replacing $\nu$ by 1 , for example, if $\sigma \in H^{2 n+2}\left(Z_{m}, Z\right)$ then

$$
\left[\theta_{k}^{*} \sigma\right]=\sigma\left(\Lambda^{2 n+2}\right)^{*}=\sigma\left(J_{2}\right)^{n+1}=\left[J_{2}^{n+1}(1)\right] \sigma .
$$

Performing this evaluation we see that:

$$
J_{2}^{n+1}(1)=k^{n+1}
$$

so that $\theta_{k}^{*}: H^{2 n+2}\left(Z_{m} ; Z\right)$ is multiplication by $\left[k^{n+1}\right]$ where $\left[k^{n+1}\right]$ denotes the equivalence class of $k^{n+1} \bmod m\left(\right.$ since $\left.H^{2 n+2}\left(Z_{m} ; Z\right) \approx Z_{m}\right)$. So we have $\theta_{k} \in E q\left(\sum^{2 n+1} / Z_{m}\right) \leftrightarrow k^{n+1} \equiv \pm 1(\bmod m)$ using Theorem 1.4. This recovers the stated result of Olum.

2B. $E q(X) \neq 0$. In studying the general problem of $E q(X)$, where $X$ is a topological space, it appears to be unknown exactly when $E q(X)=0$ or in fact what this condition would imply about the space $X$. It is known for example that if $X$ is contractible or $X=K\left(Z_{2}, n\right)$ then $E q(X)=0$. However, it appears that no other general conditions are known. However, for the spaces in this paper we have:

Theorem 2B. 1. $E q(X) \neq 0$ for all $X$ satisfying the hypotheses in $\S 1$.

Proof. If $\pi_{1}(X)$ is non-abelian then since $E q(X) \supseteqq \vartheta_{n n}\left(\pi_{1}(X)\right)$ by Corollary 1.6 and $\vartheta_{n n}\left(\pi_{1}(X)\right) \neq 0$ we get the desired result.

If $\pi_{1}(X)$ is abelian then $\pi_{1}(X)$ is a direct sum of cyclic groups of prime power order. Using Theorem 1.8 we can conclude that $E q(X)$ is either the direct sum of the corresponding self-equivalence 
groups for the summands (as in Case 1 of Theorem 8) or the direct sum of the self-equivalence groups corresponding to the summands which are two-torsion free. So we must in fact show if we look at any of these cyclic summands of $\pi_{1}(X)$, the corresponding group of self-equivalences is nonzero.

Let $p$ be a prime dividing the order of $\pi_{1}(X)$ such that $Z_{p i}$ is a direct summand of $\pi_{1}(X)$ where either $p$ is an odd prime or $i>2$. We can find such a summand since the order of $\pi_{1}(X)$ is greater than two. Now by the results of $E q\left(\Sigma^{2 k+1} / Z_{p i}\right)$ in Theorem 2A. 1 we know $E q\left(\sum^{2 k+1} / Z_{p i}\right)$ is always nonzero since there are always at least two distinct solutions to:

$$
x^{k+1} \equiv \pm 1 \quad\left(\bmod p^{i}\right)
$$

namely $x=1$ and $x=p^{i}-1$. Hence $E q(X)$ must be nontrivial since it is the direct sum of nontrivial abelian groups.

2C. The quaternionic group $Q=\{ \pm 1, \pm i, \pm j, \pm k\}$. Let $Q$ denote the eight element quaternionic group. $Q$ acts on $S^{3}$ without fixed points as a subgroup of $S^{3}$ considered as quaternions of unit norm. We have,

THEOREM 2C. 1. $E q\left(S^{3} / Q\right) \approx S_{4}$ the group of permutation of 4 symbols.

Proof. Firstly we note Aut $(Q) \approx S_{4}$ (Zassenhaus [13]). In fact in the cited reference for this fact, Aut $(Q)$ can be represented as a group of permutations of the cosets of a subgroup of order 6 contained in it. Using this representation and a projective resolution (Cartan-Eilenberg [2], p. 25) we directly calculate the induced map on cohomology of an automorphism. The calculation, which is straight forward but tedious may be found in [10]. There it is shown that in fact each automorphism induces the identity map on cohomology in the appropriate dimension, so $E q\left(S^{3} / Q\right) \approx S_{4}$.

2D. The group $D_{12}$. Let $D_{12}$ be the 12-element-group generated by elements $x, y$ subject to the relations $x^{3}=y^{2}=(x y)^{2}$ and $x y x=y . \quad D_{12}$ is a generalized quaternionic group and is seen to act on $S^{3}$ without fixed points (Milnor [4]). In a similar manner as in $2 \mathrm{C}$ (see [10]) one can show:

Theorem 2D. 1. $E q\left(S^{3} / D_{12}\right) \approx Z_{2} \times S_{3}$.

2.E. The symmetric group $S_{3}$. It is known, [5], that $S_{3}$, the 
symmetric group on three symbols, cannot act on any $n$-sphere without fixed points. However, it can be shown that it can act on a homotopy 3-sphere without fixed points [11]. We have,

THEOREM 2E. 1. $E q\left(\Sigma^{3} / S_{3}\right) \approx S_{3}$.

Proof. Since $\vartheta_{n n}\left(S_{3}\right) \approx$ Aut $\left(S_{3}\right) \approx S_{3}$ the result follows from Corollary 1.6.

In fact any periodic group can act on some homotopy sphere without fixed points [11], and so this furnishes a whole collection of examples of the type of complexes considered in this paper, which are not manifolds.

\section{REFERENCES}

1. Martin Arkowitz and C. Curjel, Groups of Homotopy Classes, Springer-Verlag, 41964.

2. Henri Cartan and Samuel Eilenberg, Homological Algebra, Princeton University Press, 1956.

3. Donald Kahn, The group of homotopy equivalences, Math. Zeitschrift, 84 (1964), 1-8.

4. Saunders Maclane, Homology, Academic Press, 1963.

5. John Milnor, Groups which act on $S^{n}$ without fixed points, Amer. J. Math., 79 (1957), 623-630.

6. Paul Olum, Mappings of manifolds and the notion of degree, Annals of Math., 58 (1953), 458-486.

7. - Self-equivalences of pseudo-projective planes, Topology, 4 (1965), 109-127.

8. J. Rutter, Groups of self-equivalences of induced spaces, Commentarii Math. Helv., 45 (1970), 236-255.

9. W. Shih, On the group $E[X]$ of homotopy equivalence maps, Bull. Amer. Math. Soc., (1964), 492.

10. D. Smallen, The Group of Self-equivalences of Certain Manifolds, Thesis, The University of Rochester 1972.

11. R. G. Swan, Periodic resolutions for finite groups, Ann. of Math., 72 (1960), 267-291.

12. E. Weiss, Cohomology of Groups, Academic Press, 1969.

13. H. Zassenhaus, The Theory of Groups, Chelsea, 1956.

Received March 9, 1973 and in revised form July 13, 1973. The author wishes to thank his advisor, Professor Norman Stein, and the referee, for comments which led to a shorter and more general exposition of this work.

UNIVERSITY OF ROCHESTER

AND

HAMILTON COLLEGE 


\section{PACIFIC JOURNAL OF MATHEMATICS}

\section{EDITORS}

RICHARD ARENS (Managing Editor)

University of California

Los Angeles, California 90024

R. A. Beaumont

University of Washington

Seattle, Washington 98105
J. DugundJI

Department of Mathematics University of Southern California Los Angeles, California 90007

D. Gilbarg AND J. Milgram Stanford University

Stanford, California 94305

\section{ASSOCIATE EDITORS}
E. F. BECKENBACH
B. H. NeUmanN
F. WOLF
K. YOSHIDA

\section{SUPPORTING INSTITUTIONS}

UNIVERSITY OF BRITISH COLUMBIA CALIFORNIA INSTITUTE OF TECHNOLOGY

UNIVERSITY OF CALIFORNIA

MONTANA STATE UNIVERSITY

UNIVERSITY OF NEVADA

NEW MEXICO STATE UNIVERSITY

OREGON STATE UNIVERSITY

UNIVERSITY OF OREGON

OSAKA UNIVERSITY
UNIVERSITY OF SOUTHERN CALIFOF

STANFORD UNIVERSITY

UNIVERSITY OF TOKYO

UNIVERSITY OF UTAH

WASHINGTON STATE UNIVERSITY UNIVERSITY OF WASHINGTON

AMERICAN MATHEMATICAL SOCIET NAVAL WEAPONS CENTER 


\section{Pacific Journal of Mathematics}

\section{Vol. 54, No. 1 \\ May, 1974}

Ralph K Amayo, Engel Lie rings with chain conditions ..................

Bernd Anger and Jörn Lembcke, Hahn-Banach type theorems for hypolinear

functionals on preordered topological vector spaces ..................

Gregory Frank Bachelis and Samuel Ebenstein, On $\Lambda(p)$ sets ................

Harvey Isaac Blau, Indecomposable modules for direct products of finite

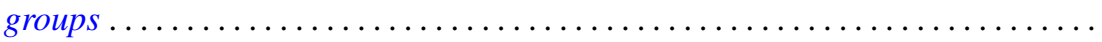

Larry Eugene Bobisud and James Calvert, Singular perturbation of a

time-dependent Cauchy problem in a Hilbert space ................

Walter D. Burgess and Robert Raphael, Abian's order relation and orthogonal

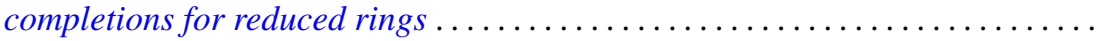

James Diederich, Representation of superharmonic functions mean continuous at

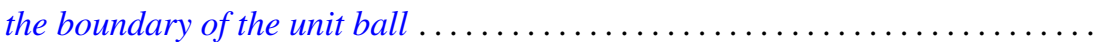

Aad Dijksma and Hendrik S. V. de Snoo, Self-adjoint extensions of symmetric

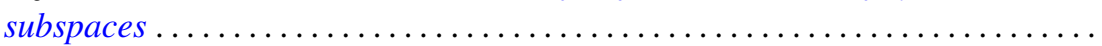

Gustave Adam Efroymson, A Nullstellensatz for Nash rings . . . . . . . . . . . . .

John D. Elwin and Donald R. Short, Branched immersions onto compact orientable surfaces . . . . . . . . . . . . . . . . . . . . . . . . .

John Douglas Faires, Comparison of the states of closed linear

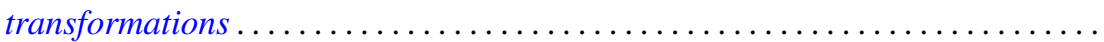

Joe Wayne Fisher and Robert L. Snider, On the von Neumann regularity of rings with regular prime factor rings .

Franklin Takashi Iha, A unified approach to boundary value problems on compact intervals

Palaniappan L. Kannappan and Che Tat $\mathrm{Ng}$, On functional equations connected with directed divergence, inaccuracy and generalized directed divergence

Samir A. Khabbaz and Elias Hanna Toubassi, The module structure of Ext $(F, T)$ over the endomorphism ring of $T$...

Garo K. Kiremidjian, On deformations of complex compact manifolds with boundary.

Dimitri Koutroufiotis, Mappings by parallel normals preserving principal

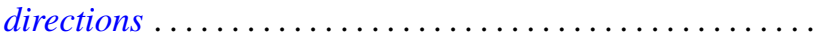

W. K. Nicholson, Semiperfect rings with abelian adjoint group

Norman R. Reilly, Extension of congruences and homomorphisms to translational hulls

Sadahiro Saeki, Symmetric maximal ideals in $M(G)$

Brian Kirkwood Schmidt, On the homotopy invariance of certain functors ...

H. J. Shyr and T. M. Viswanathan, On the radicals of lattice-ordered rings ...

Indranand Sinha, Certain representations of infinite group algebras ...

David Smallen, The group of self-equivalences of certain complexes ...

Kalathoor Varadarajan, On a certain problem of realization in homotopy

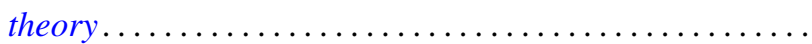

